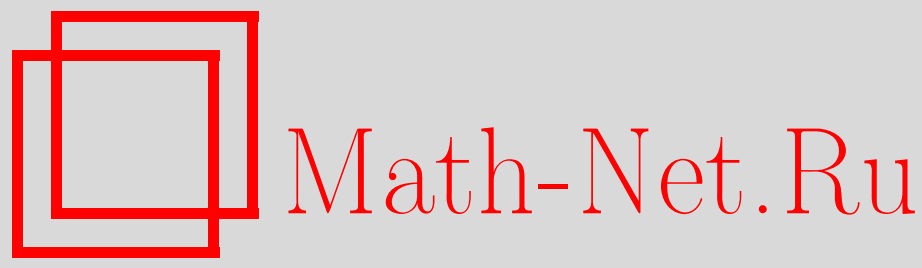

А. А. Гриб, Ю. В. Павлов, Энергия столкновений частиц в эргосфере вращающихся черных дыр, ТМФ, 2013, том 176, номер 1, 60-68

DOI: https://doi.org/10.4213/tmf8480

Использование Общероссийского математического портала Math-Net.Ru подразумевает, что вы прочитали и согласны с пользовательским соглашением http://www.mathnet.ru/rus/agreement

Параметры загрузки:

IP : 52.6 .47 .48

26 апреля 2023 г., 13:46:35

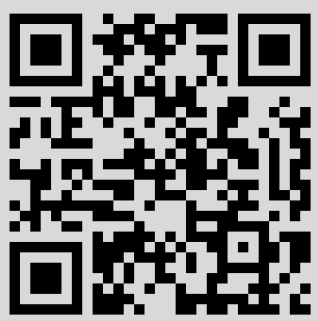




\section{ЭНЕРГИЯ СТОЛКНОВЕНИЙ ЧАСТИЦ В ЭРГОСФЕРЕ ВРАЩАЮЩИХСЯ ЧЕРНЫХ ДЫР}

Приведены новые оценки возможности появления резонанса в системе центра масс при столкновении частиц в эргосферах вращающихся черных дыр активных ядер галактик. Исследован новый эффект - рост энергии в системе центра масс в зависимости от углового момента частицы, возможный в любой точке эргосферы.

Ключевые слова: черные дыры, метрика Керра, столкновения частиц, геодезические.

DOI: $10.4213 / \operatorname{tmf} 8480$

\section{1. ВВЕДЕНИЕ}

Активные ядра галактик согласно общему мнению являются вращающимися черными дырами, описываемыми метрикой Керра. Метрика Керра, в отличие от метрики Шварцшильда, предсказывает существование особой области вне горизонта черной дыры - эргосферы. В эргосфере элементарные частицы не могут не вращаться вместе с черной дырой. Последнее приводит к тому, что эта область является естественным суперколлайдером элементарных частиц. В работе [1] для экстремальных керровских черных дыр было обнаружено существование особого резонанса при столкновении двух частиц вблизи горизонта. При этом энергия в системе центра масс могла неограниченно расти. В наших работах [2]-[4] существование подобного резонанса было показано в случае двукратного рассеяния для неэкстремальных черных дыр - реальных активных ядер галактик.

В настоящей работе мы обобщаем наши результаты для резонанса, получив некоторые новые условия его возникновения, а также учитываем возможность отрицательных энергий частиц, допустимую в эргосфере. Оценивается время достижения планковской энергии в системе центра масс. Кроме того, обнаружен новый эффект

${ }^{*}$ Российский государственный педагогический университет им. А. И. Герцена, Санкт-Петербург, Россия. E-mail: andrei_grib@mail.ru

†Лаборатория теоретической физики им. А. А. Фридмана, Санкт-Петербург, Россия

${ }^{\ddagger}$ Институт проблем машиноведения РАН, Санкт-Петербург, Россия.

E-mail: yuri.pavlov@mail.ru 
роста энергии в зависимости от углового момента частицы в эргосфере, возможный, в отличие от резонанса, в любой точке эргосферы.

В статье используется система единиц, в которой гравитационная постоянная и скорость света равны единице: $G=c=1$.

\section{2. ЯВЛЕНИЕ РЕЗОНАНСА ПРИ СТОЛКНОВЕНИИ ЧАСТИЦ ВБЛИЗИ ГОРИЗОНТА}

Метрика Керра вращающейся черной дыры [5] в координатах Бойера-Линдквиста [6] имеет вид

$$
\begin{aligned}
d s^{2}=d t^{2} & -\frac{2 M r\left(d t-a \sin ^{2} \theta d \varphi\right)^{2}}{r^{2}+a^{2} \cos ^{2} \theta}- \\
& -\left(r^{2}+a^{2} \cos ^{2} \theta\right)\left(\frac{d r^{2}}{\Delta}+d \theta^{2}\right)-\left(r^{2}+a^{2}\right) \sin ^{2} \theta d \varphi^{2},
\end{aligned}
$$

где

$$
\Delta=r^{2}-2 M r+a^{2},
$$

$M$ - масса черной дыры, $J=a M=A M^{2}$ - ее момент импульса. Полагаем, что направление оси вращения черной дыры соответствует $\theta=0$, т. е. $A \geqslant 0$. Горизонт событий керровской дыры соответствует значению координаты

$$
r=r_{\mathrm{H}} \equiv M+\sqrt{M^{2}-a^{2}} .
$$

Поверхность предела статичности определяется значением

$$
r=r_{1} \equiv M+\sqrt{M^{2}-a^{2} \cos ^{2} \theta} .
$$

В случае $A \leqslant 1$ область пространства-времени между пределом статичности и горизонтом событий называется эргосферой.

Уравнения экваториальных геодезических в метрике Керра имеют вид [7]

$$
\begin{aligned}
\frac{d t}{d \lambda} & =\frac{1}{\Delta_{x}}\left[\left(x^{2}+A^{2}+\frac{2 A^{2}}{x}\right) E-\frac{2 A}{x} j\right], \\
\frac{d \varphi}{d \lambda} & =\frac{1}{M \Delta_{x}}\left[\frac{2 A}{x} E+\left(1-\frac{2}{x}\right) j\right], \\
\left(\frac{d r}{d \lambda}\right)^{2} & =E^{2}+\frac{2}{x^{3}}(A E-j)^{2}+\frac{A^{2} E^{2}-j^{2}}{x^{2}}-\frac{\Delta_{x}}{x^{2}} m^{2},
\end{aligned}
$$

где $x=r / M, \Delta_{x}=x^{2}-2 x+A^{2}, E=\mathrm{const}-$ энергия движущейся частицы, $j M=$ const - проекция ее момента импульса на ось вращения черной дыры, $m$ - масса покоя движущейся частицы. Для частицы с $m \neq 0$ параметр $\lambda=\tau / m$, где $\tau$ - ее собственное время.

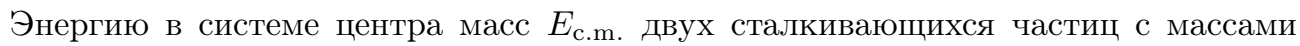
покоя $m_{1}$ и $m_{2}$ найдем, возведя в квадрат формулу

$$
\left(E_{\text {c.m. }}, 0,0,0\right)=p_{(1)}^{i}+p_{(2)}^{i},
$$

где $p_{(n)}^{i}-4$-импульсы частиц $(n=1,2)$. Поскольку $p_{(n)}^{i} p_{(n) i}=m_{n}^{2}$, то

$$
E_{\mathrm{c} . \mathrm{m} .}^{2}=m_{1}^{2}+m_{2}^{2}+2 p_{(1)}^{i} p_{(2) i} .
$$


Для частиц, свободно падающих в экваториальной плоскости вращающейся черной дыры, с энергиями $E_{1}$ и $E_{2}$ (относительно бесконечности) и моментами импульса $j_{1}, j_{2}$ из уравнений (5) получим

$$
\begin{aligned}
p_{(1)}^{i} p_{(2) i}= & \frac{1}{x \Delta_{x}}\left\{E_{1} E_{2}\left[x^{3}+A^{2}(x+2)\right]-2 A\left(j_{1} E_{2}+j_{2} E_{1}\right)+\right. \\
& +j_{1} j_{2}(2-x)-\sqrt{2 E_{1}^{2} x^{2}+2\left(j_{1}-E_{1} A\right)^{2}-j_{1}^{2} x+\left(E_{1}^{2}-m_{1}^{2}\right) x \Delta_{x}} \times \\
& \left.\times \sqrt{2 E_{2}^{2} x^{2}+2\left(j_{2}-E_{2} A\right)^{2}-j_{2}^{2} x+\left(E_{2}^{2}-m_{2}^{2}\right) x \Delta_{x}}\right\} .
\end{aligned}
$$

Знаменатель формулы обращается в нуль на горизонте событий. Найдя предел данного выражения при $r \rightarrow r_{\mathrm{H}}$, из (7) получим [8]

$$
E_{\text {c.m. }}^{2}\left(r \rightarrow r_{\mathrm{H}}\right)=\frac{\left(j_{1 \mathrm{H}} j_{2}-j_{2 \mathrm{H}} j_{1}\right)^{2}}{4\left(j_{1 \mathrm{H}}-j_{1}\right)\left(j_{2 \mathrm{H}}-j_{2}\right)}+m_{1}^{2}\left[1+\frac{j_{2 \mathrm{H}}-j_{2}}{j_{1 \mathrm{H}}-j_{1}}\right]+m_{2}^{2}\left[1+\frac{j_{1 \mathrm{H}}-j_{1}}{j_{2 \mathrm{H}}-j_{2}}\right],
$$

где

$$
j_{n \mathrm{H}}=\frac{2 E_{n} x_{\mathrm{H}}}{A}=\frac{E_{n}}{M \Omega_{\mathrm{H}}},
$$

$x_{\mathrm{H}}=r_{\mathrm{H}} / M, \Omega_{\mathrm{H}}=A / 2 r_{\mathrm{H}}-$ "угловая скорость" вращения горизонта событий [9]. Формула (9) применима и для случаев, когда одна или обе частицы безмассовые.

Как следует из (9), энергия столкновения в системе центра масс двух падающих частиц неограниченно возрастает вблизи горизонта, если одна из частиц имеет момент импульса $j_{\mathrm{H}}$ (критическая частица), а у другой $j<j_{\mathrm{H}}$. На это явление (для случая $A=1$ и $E=m$ ) было впервые указано в работе [1]. Позднее, в работах [10], [11] отмечалось, что в случае $A<1$ такой резонанс недостижим для падающей на черную дыру из бесконечности частицы. В частности, если черная дыра имеет угловой момент, равный торновскому пределу для астрофизической черной дыры $(A \approx 0.998)$ (см. [12]), то $E_{\text {c.m. }}^{\max } / \sqrt{m_{1} m_{2}} \approx 18.97$. Однако в работе [2] нами было показано, что в случае промежуточных столкновений вблизи горизонта событий с третьей частицей падающая частица может приобрести момент импульса (также это возможно и при распаде исходной частицы, для одного из осколков), близкий к критическому, и впоследствии энергия ее столкновения с другой свободно падающей частицей может стать сколь угодно большой. Далее будем называть такую ситуацию механизмом промежуточных столкновений. В работе [13] нами была найдена причина появления очень больших энергий столкновения вблизи горизонта. Для массивных частиц картина столкновения с неограниченно большой энергией в системе центра масс оказывается следующей. Критическая частица имеет в окрестности горизонта нулевую радиальную составляющую скорости $d r / d \tau=0$. Сталкивающаяся с ней некритическая частица при движении к горизонту, наоборот, ускоряется так, что их относительная скорость приближается к скорости света, что и приводит к неограниченному возрастанию энергии столкновения. Для черных дыр других типов это явление рассмотрено в работах Заславского [14]-[16].

Докажем далее, что для достижения неограниченно больших энергий столкновения частиц, падающих на черную дыру, механизм промежуточных столкновений необходим не только для неэкстремальных $A<1$ черных дыр, но и в экстремальном 
случае, при определенных энергиях массивных частиц. Также покажем, что энергия столкновения в эргосфере может быть неограниченно велика и для некритических частиц в случае больших по абсолютной величине, но отрицательных значений проекции момента импульса одной из частиц.

\section{3. УСЛОВИЯ ОСУЩЕСТВЛЕНИЯ РЕЗОНАНСА}

Рассмотрим подробнее движение частиц в экваториальной плоскости вращающейся черной дыры с $A \leqslant 1$. Допустимые значения момента импульса могут быть найдены из уравнений (5) в соответствии с условием $(d r / d \lambda)^{2} \geqslant 0$ и требованием движения "вперед по времени" [17]: $d t / d \lambda>0$.

При $x>2$, т. е. вне эргосферы, эти требования приводят к следующим ограничениям:

$$
E \geqslant \sqrt{\frac{x-2}{x}} m, \quad j \in\left[j_{-}, j_{+}\right], \quad j_{ \pm}=\frac{-2 A E \pm x \sqrt{\Delta_{x}\left(E^{2}-(x-2) m^{2} / x\right)}}{x-2} .
$$

Граничные значения $j_{ \pm}$соответствуют условию $d r / d \lambda=0$. На границе эргосферы

$$
x=2 \Rightarrow E \geqslant 0, \quad j \leqslant E\left(A+\frac{2}{A}\right)-\frac{A m^{2}}{2 E} .
$$

Внутри эргосферы

$$
x_{\mathrm{H}}<x<2 \Rightarrow j \leqslant j_{-}(x)=\frac{2 A E-x \sqrt{\Delta_{x}\left(E^{2}+(2-x) m^{2} / x\right)}}{2-x} .
$$

Отсюда, в частности, следует, что при отрицательной энергии $E$ частицы в эргосфере проекция ее момента импульса на ось вращения черной дыры также должна быть отрицательной. Заметим, однако, что при этом вращение в координатах Бойера-Линдквиста для любых частиц в эргосфере будет происходить в сторону вращения черной дыры (явление “увлечения тел вращающейся черной дырой” [9]). Действительно, для времениподобных геодезических условие $d s^{2}>0$ требует выполнения неравенства $d \varphi / d t>0$, так как остальные слагаемые в выражении для квадрата интервала в эргосфере, как видно из (1), отрицательны. Поэтому утверждение "только частицы, направление вращения которых вокруг черной дыры противоположно вращению самой черной дыры, могут иметь отрицательную энергию”, приведенное в фундаментальной монографии [7], неверно.

Отметим также, что энергия столкновения в системе центра масс всегда положительна (даже в случае отрицательных значений $E_{1}, E_{2}$ ) и удовлетворяет условию

$$
E_{\text {c.m. }} \geqslant m_{1}+m_{2} \text {. }
$$

Это следует из того, что в системе центра масс сталкивающиеся частицы движутся друг к другу с некоторыми скоростями. Неравенство (14) также может быть получено и в координатах Бойера-Линдквиста из формул (7), (8) путем достаточно громоздких алгебраических преобразований, при учете ограничений (11)-(13).

При стремлении точки $r$ к горизонту $r_{\mathrm{H}}$ получаем из (13) ограничение

$$
x \rightarrow x_{\mathrm{H}} \Rightarrow j \leqslant j_{\mathrm{H}} \equiv \frac{2 E x_{\mathrm{H}}}{A} .
$$

Таким образом, при заданной энергии "критическая" частица имеет максимально возможный у горизонта событий момент импульса. 
Исследуем поведение функции $j_{-}(x)$ в окрестности горизонта. Для значения производной в пределе $x \rightarrow x_{\mathrm{H}}+0$ получим

$$
\begin{aligned}
& A<1 \Rightarrow j_{-}^{\prime}(x) \sim-\frac{\left(x_{\mathrm{H}}-1\right) \sqrt{E^{2} x_{\mathrm{H}}^{2}+m^{2} A^{2}}}{x_{\mathrm{C}} \sqrt{\left(x_{\mathrm{H}}-x_{\mathrm{C}}\right)\left(x-x_{\mathrm{H}}\right)}} \rightarrow-\infty, \quad x \rightarrow x_{\mathrm{H}}, \\
& A=1 \Rightarrow j_{-}^{\prime}\left(x_{\mathrm{H}}\right)=2 E-\sqrt{E^{2}+m^{2}}
\end{aligned}
$$

где $x_{\mathrm{C}}=1-\sqrt{1-A^{2}}$. Для частицы в поле экстремально вращающейся черной дыры из (17) получим

$$
A=1 \Rightarrow \begin{cases}j_{-}^{\prime}\left(x_{\mathrm{H}}\right)>0, & E>\frac{m}{\sqrt{3}} \\ j_{-}^{\prime}\left(x_{\mathrm{H}}\right)<0, & E<\frac{m}{\sqrt{3}} .\end{cases}
$$

Разрешенная зона для частиц с критическим моментом импульса $2 E>0$ в экстремально вращающейся черной дыре определяется условием

$$
\left(\frac{d r}{d \lambda}\right)^{2}=\frac{(x-1)^{2}}{x^{3}}\left[2 E^{2}+x\left(E^{2}-m^{2}\right)\right] \geqslant 0
$$

т. е. допустимы любое $x$ при $E \geqslant m$ и некоторая область вне горизонта

$$
x \leqslant x_{E}=\frac{2 E^{2}}{m^{2}-E^{2}}
$$

для массивных частиц с $1 / \sqrt{3}<E / m<1$.

Таким образом, окрестность вне горизонта для частицы с критическим значением момента импульса является запрещенной зоной в случае черной дыры с $A<1$ при любой энергии, а для экстремально вращающейся черной дыры $A=1$ при энергии частицы $E \leqslant m / \sqrt{3}$. В этих случаях неограниченно большая энергия столкновения может быть реализована только за счет механизма промежуточных столкновений (см. также [18]).

Если частица с энергией $E_{1}$ и критическим значением момента импульса $j_{1 \mathrm{H}}=$ $2 E_{1}$ падает на экстремально вращающуюся черную дыру из бесконечности или (при $1 / \sqrt{3}<E / m<1)$ из указанной в $(20)$ области и сталкивается с другой свободно падающей некритической частицей (с энергией $E_{2}$ и моментом импульса $j_{2}$ ) в некоторой точке с радиальной координатой $r$ вблизи горизонта, то энергия столкновения в системе центра масс, в соответствии с (7), (8) равная

$$
\begin{aligned}
E_{\mathrm{c} . \mathrm{m} .}^{2}= & m_{1}^{2}+m_{2}^{2}+\frac{2}{x(x-1)}\left[E_{1} E_{2}\left(x^{2}+x+2\right)-2 E_{1} j_{2}-\sqrt{E_{1}^{2}(2+x)-m_{1}^{2} x} \times\right. \\
& \left.\times \sqrt{\left(E_{2}^{2}(2+x)-m_{2}^{2} x\right)(x-1)^{2}+\left(2 E_{2}-j_{2}\right)\left(2 E_{2} x-j_{2}(2-x)\right)}\right]
\end{aligned}
$$

неограниченно возрастает при $r \rightarrow r_{\mathrm{H}}$. 


\section{4. ЭНЕРГИЯ СТОЛКНОВЕНИЯ С ЧАСТИЦЕЙ С БОЛЬШИМ МОМЕНТОМ ИМПУЛЬСА}

Рассмотрим другую возможность для столкновений в эргосфере вращающейся черной дыры со сколь угодно большой энергией в системе центра масс при фиксированных энергиях частиц относительно бесконечности. Для этого заметим, что при фиксированной энергии частицы ее проекция момента импульса может быть в эргосфере отрицательной и сколь угодно большой по абсолютной величине в соответствии с ограничениями (12), (13). Найдя асимптотику выражения (8) при $j_{2} \rightarrow-\infty$ и фиксированном $r$ в эргосфере, получим

$$
\begin{aligned}
E_{\mathrm{c} . \mathrm{m} .}^{2} \approx & \frac{-2 j_{2}}{x \Delta_{x}}\left[2 A E_{1}-j_{1}(2-x)-\right. \\
& \left.\quad-\sqrt{2-x} \sqrt{2 E_{1}^{2} x^{2}+2\left(j_{1}-E_{1} A\right)^{2}-j_{1}^{2} x+\left(E_{1}^{2}-m_{1}^{2}\right) x \Delta_{x}}\right] .
\end{aligned}
$$

Эта асимптотическая формула верна для любых допустимых $E_{1}, j_{1}$ (см. (13)) при $r_{\mathrm{H}}<r<2$ и для $E_{1}>0$ и $j_{1}$, удовлетворяющем (12), при $r=2$. Заметим, что выражение в квадратных скобках в (22) положительно в эргосфере. Это очевидно для $x=2$ и следует из ограничений (13) при $x_{\mathrm{H}}<x<2$, поскольку $2 A E=\left(j_{+}+\right.$ $\left.j_{-}\right)(2-x)$ и

$$
\sqrt{2 E^{2} x^{2}+2(j-E A)^{2}-j^{2} x+\left(E^{2}-m^{2}\right) x \Delta_{x}}=\sqrt{(2-x)\left(j_{+}-j_{1}\right)\left(j_{-}-j_{1}\right)} .
$$

Таким образом, как следует из (22), при падении частиц, на вращающуюся черную дыру в экваториальной плоскости в любой точке эргосферы возможсны столкновения частии, со сколь угодно большой энергией в системе иентра масс при $j_{2} \rightarrow-\infty$ u фиксированных энергиях частии $E_{1}, E_{2}$. Энергия столкновения в системе центра масс возрастает пропорционально $\sqrt{\left|j_{2}\right|}$.

Отметим, что большие отрицательные значения проекции момента импульса запрещены при фиксированной энергии частицы вне эргосферы. Поэтому такие столкновения не осуществляются для частиц, падающих из бесконечности. Если же частица уже попала в эргосферу и там в результате взаимодействия с другими частицами приобрела большое отрицательное значение проекции момента импульса (не требуется приобретения больших энергий!), то ее последующее столкновение с падающей в черную дыру частицей может иметь очень большую энергию в системе центра масс.

При больших значениях $-j_{2}$ энергия столкновения вблизи горизонта в системе центра масс в соответствии с (9) равна

$$
E_{\mathrm{c} . \mathrm{m} .} \approx \sqrt{-j_{2} \frac{j_{1 \mathrm{H}}^{2}+4 m_{1}^{2}}{4\left(j_{1 \mathrm{H}}-j_{1}\right)}}
$$

В зависимости от значения момента импульса $j_{1}$ она может быть как больше, так и меньше, чем при столкновении в других точках эргосферы.

Приведем оценки значений моментов импульса, необходимых для высокоэнергетических столкновений. В системе СИ выражение для критического значения момента импульса (10) имеет вид

$$
J_{\mathrm{H}}=j_{\mathrm{H}} M c=\frac{G M}{c^{3}} \frac{2 E x_{\mathrm{H}}}{A} .
$$

3 Теоретическая и математическая физика, т. 176, № 1, 2013 г. 
При $E=m c^{2}, A \approx 1$ и массе черной дыры, равной массе Солнца, таким моментом импульса обладала бы, например, частица, вращающаяся вокруг черной дыры на расстоянии, равном расстоянию Земли от Солнца, со скоростью порядка 6 м/с. Если бы частица в эргосфере при $x \approx 2$ имела бы тот же момент импульса, как у частицы, вращающейся на орбите Земли $r \approx 1.5 \cdot 10^{11}$ м с орбитальной скоростью Земли $v \approx 3 \cdot 10^{4} \mathrm{M} / \mathrm{c}$, то при столкновении с частицей с $E_{1}=m_{1} c^{2}$ энергия столкновения составила бы, в соответствии с формулой $(22)$, порядка $10^{2} \sqrt{m_{1} m_{2}} c^{2}$. При энергии столкновения $10^{9} \sqrt{m_{1} m_{2}} c^{2}$ и скорости частицы $v \approx 10^{3} \mathrm{M} / \mathrm{c}$ требуемый момент импульса соответствовал бы вращению на расстоянии от звезды порядка размера горизонта расширяющейся Вселенной $\left(\approx 10^{26} \mathrm{M}\right)$. Такой момент импульса относительно Солнца имеют частицы сверхвысоких энергий $10^{19}-10^{20}$ эВ, наблюдаемые в космических лучах на Земле.

Эти оценки показывают, что достижение сверхвысоких энергий при столкновении частиц обычных масс (например, протонов) в таком механизме представляется нереалистическим. Для этого потребовались бы многократные столкновения с приобретением каждый раз дополнительного отрицательного момента импульса. Однако частицы с массой порядка масштаба теории Великого Объединения (возможно, такие частицы присутствуют в темной материи [19]) могут приобрести энергию порядка планковской.

\section{5. ВРЕМЯ ДОСТИЖЕНИЯ СВЕРХВЫСОКИХ ЭНЕРГИЙ ДЛЯ РЕАЛЬНЫХ ЧЕРНЫХ ДЫР}

Значения момента импульса, близкие к $J_{\mathrm{L}}$, могут иметь место для свободно падающих на черную дыру частиц. Так, нерелятивистская на бесконечности частица $(E=m)$ достигает горизонта событий при падении на черную дыру, если ее момент импульса $j$ лежит в интервале $j_{\mathrm{L}} \leqslant j \leqslant j_{\mathrm{R}}$, где

$$
j_{\mathrm{L}}=-2 m[1+\sqrt{1+A}], \quad j_{\mathrm{R}}=2 m[1+\sqrt{1-A}] .
$$

При $A \rightarrow 1$ имеет место $j_{\mathrm{R}} \rightarrow j_{\mathrm{H}}$. В случае $A=1$ имеем $j_{\mathrm{R}}=j_{\mathrm{H}}=2 m$. Однако в этом случае время, необходимое для осуществления столкновения с большой энергией, очень велико. Количественные оценки даны, например, в работах [3], [4]. Чтобы два протона столкнулись с энергией порядка характерного масштаба теории Великого Объединения, понадобилось бы время $10^{24}$ с, которое превышает возраст Вселенной. Для получения неограниченно большой энергии столкновения в случае экстремально вращающихся черных дыр $(A=1)$ нужен бесконечно большой интервал как координатного $\Delta t \sim E_{\text {c.m. }}^{2}$, так и собственного времени $\Delta \tau \sim \ln E_{\text {c.m. }}$ падения частицы.

Для столкновений при падении на вращающуюся черную дыру с $A<1$ в рамках механизма промежуточных столкновений с приобретением вблизи горизонта событий момента импульса, очень близкого к $j_{\mathrm{H}}$, оценки времени падения частиц с расстояния до черной дыры, равного нескольким $r_{\mathrm{H}}$ (порядка расстояния от аккреционного диска), представлены в работах [8], [13]:

$$
\Delta t \sim \frac{4 M x_{\mathrm{H}}}{\sqrt{1-A^{2}}} \ln \frac{E_{\mathrm{c} . \mathrm{m} .}}{\sqrt{m_{1} m_{2}}} .
$$


Так, для $A=0.998$ имеем

$$
\Delta t \sim 3.2 \cdot 10^{-4} \frac{M}{M_{\odot}} \ln \frac{E_{\text {c.m. }}}{\sqrt{m_{1} m_{2}}} \mathrm{c} .
$$

Взяв значение энергии Великого Объединения $E_{\mathrm{c} . \mathrm{m}} / \sqrt{m_{1} m_{2}}=10^{14}$ и значение массы черной дыры $10^{8} M_{\odot}$, типичное для активных ядер галактик, получим $\Delta t \sim 10^{6}$ с, что значительно меньше, чем в экстремальном случае. Поскольку зависимость времени от энергии логарифмическая, для планковского значения энергии столкновения $\sim 10^{19}$ ГэВ понадобилось бы большее время, но того же порядка величины.

Что касается времени обратного движения частиц (продуктов столкновения) с очень высокой энергией из окрестностей горизонта, например, к Земле, то с учетом обратимости уравнений движения во времени легко видеть, что время движения частиц до аккреционного диска составит порядка 10-15 дней, а далее необходимо длительное время для движения от астрофизических черных дыр до Земли.

\section{6. ЗАКЛЮЧЕНИЕ}

В заключение обсудим возможный физический смысл полученных результатов. Активные ядра галактик, возможно, являются источниками частиц космических лучей сверхвысоких энергий, наблюдаемых в последние годы [20], [21]. Кроме того, они характеризуются джетами, природа которых до конца не ясна. Поэтому существование в эргосфере естественного и, по-видимому, самого мощного во Вселенной суперколлайдера должно приводить к наблюдаемым астрофизическим следствиям. Наконец, возможность достижения планковских значений энергии позволяет проверить различные гипотезы квантовой гравитации.

Благодарности. Исследование проведено в рамках сотрудничества с Copernicus Center of Interdisciplinary Studies (Краков, Польша) и поддержано The John Templeton Foundation.

\section{Список литературы}

[1] M. Bañados, J. Silk, S. M. West, Phys. Rev. Lett., 103:11 (2009), 111102, 4 pp.

[2] А. A. Grib, Yu. V. Pavlov, Писъма в ЖЖЭТФ, 92:3 (2010), 147-151.

[3] A. A. Grib, Yu. V. Pavlov, Astropart. Phys., 34:7 (2011), 581-586.

[4] A. A. Grib, Yu. V. Pavlov, Grav. Cosmol., 17:1 (2011), 42-46.

[5] R. P. Kerr, Phys. Rev. Lett., 11:5 (1963), 237-238.

[6] R. H. Boyer, R. W. Lindquist, J. Math. Phys., 8:2 (1967), 265-281.

[7] С. Чандрасекар, Математическая теория черных дыр, Мир, М., 1986.

[8] A. A. Grib, Yu. V. Pavlov, O.F. Piattella, Grav. Cosmol., 18:1 (2012), 70-75, arXiv: 1203.4952.

[9] И. Д. Новиков, В. П. Фролов, Физика черных дыр, Наука, М., 1986.

[10] E. Berti, V. Cardoso, L. Gualtieri, F. Pretorius, U. Sperhake, Phys. Rev. Lett., 103:23 (2009), 239001, 1 pp., arXiv: 0911.2243.

[11] T. Jacobson, T. P. Sotiriou, Phys. Rev. Lett., 104:2 (2010), 021101, 3 pp., arXiv: 0911.3363.

[12] K.S. Thorne, Astrophys. J., 191 (1974), 507-520.

[13] A. A. Grib, Yu. V. Pavlov, O. F. Piattella, Int. J. Modern Phys. A, 26:22 (2011), 3856-3867.

[14] O. B. Zaslavskii, Class. Quantum Grav., 28:10 (2011), 105010, 7 pp., arXiv: 1011.0167. 
[15] O. B. Zaslavskii, Phys. Rev. D, 84:2 (2011), 024007, 6 pp., arXiv: 1104.4802.

[16] O. B. Zaslavskii, Phys. Lett. B, 712:3 (2012), 161-164, arXiv: 1202.0565.

[17] Р. М. Уолд, Общая теория относительности, РУДН, М., 2008.

[18] T. Harada, M. Kimura, Phys. Rev. D, 83:8 (2011), 084041, 9 pp., arXiv: 1102.3316.

[19] A. A. Grib, Yu. V. Pavlov, Modern Phys. Lett. A, 23:16 (2008), 1151-1159, arXiv: 0712.2667.

[20] J. Abraham, P. Abreu, M. Aglietta et al. [The Pierre Auger Collaboration], Science, 318:5852 (2007), 938-943, arXiv: 0711.2256.

[21] P. Abreu, M. Aglietta, E. J. Ahn et al. [The Pierre Auger Collaboration], Astropart. Phys., 34:5 (2010), 314-326. 